\title{
On (co)pure Baer injective modules
}

\author{
M. F. Hamid
}

\author{
Communicated by R. Wisbauer
}

\begin{abstract}
A Bstract. For a given class of $R$-modules $\mathcal{Q}$, a module $M$ is called $\mathcal{Q}$-copure Baer injective if any map from a $\mathcal{Q}$-copure left ideal of $R$ into $M$ can be extended to a map from $R$ into $M$. Depending on the class $\mathcal{Q}$, this concept is both a dualization and a generalization of pure Baer injectivity. We show that every module can be embedded as $\mathcal{Q}$-copure submodule of a $\mathcal{Q}$-copure Baer injective module. Certain types of rings are characterized using properties of $\mathcal{Q}$-copure Baer injective modules. For example a $\operatorname{ring} R$ is $\mathcal{Q}$-coregular if and only if every $\mathcal{Q}$-copure Baer injective $R$-module is injective.
\end{abstract}

\section{Introduction}

Let $\mathcal{Q}$ be a non-empty class of left $R$-modules. An exact sequence

$$
0 \rightarrow A \stackrel{f}{\rightarrow} B \stackrel{g}{\rightarrow} C \rightarrow 0
$$

of left $R$-modules is called $\mathcal{Q}$-copure if every module in $\mathcal{Q}$ is injective with respect to the sequence. In this case, $f$ is called a $\mathcal{Q}$-copure monomorphism and $g$ a $\mathcal{Q}$-copure epimorphism [4, p.322]. If we denote by $\mathcal{P} \mathcal{I}$ the class of pure injective modules then the $\mathcal{P} \mathcal{I}$-copure sequences are exactly the pure exact ones, see [4, p.290]. So not only does this concept dualize purity but generalizes it as well. We will need the following lemma later.

2020 MSC: 16D50.

Key words and phrases: $\mathcal{Q}$-copure submodule, $\mathcal{Q}$-copure Baer injective module, pure Baer injective module.. 
Lemma 1. [4, p.323] For a given class of modules $\mathcal{Q}$, the following hold.

1 ) Any pushout of a $\mathcal{Q}$-copure monomorphism is a $\mathcal{Q}$-copure monomorphism.

2) If $g \circ f$ is a $\mathcal{Q}$-copure monomorhphism, where $f: A \rightarrow B$ and $g: B \rightarrow C$, then so is $f$.

For details about $\mathcal{Q}$-copure submodules the reader is referred to section 38 of [4].

Thani [3] introduced pure Baer injective modules as those modules which are injective with respect to all pure exact sequences with the ring $R$ as a middle term. Here we study $\mathcal{Q}$-copure Baer injective modules for some given non-empty class of left $R$-modules $\mathcal{Q}$, i.e. modules injective with respect to all $\mathcal{Q}$-copure sequences with $R$ as a middle term. Pure Baer injective modules are, now, a special case of $\mathcal{Q}$-copure Baer injectives by choosing $\mathcal{Q}=\mathcal{P} \mathcal{I}$.

Unless otherwise stated the ring $R$ is always associative with identity, all modules are left unital $R$-modules, and $\mathcal{Q}$ is a non-empty class of modules. If there is no confusion or if the class $\mathcal{Q}$ is known we will drop the letter $\mathcal{Q}$ and just say copure sequences and copure Baer injective modules.

\section{Copure Baer injective modules}

Definition 1. An $R$-module $M$ is called $\mathcal{Q}$-copure Baer injective if any homomorphism from a $\mathcal{Q}$-copure left ideal of $R$ into $M$ has an extension to a homomorphism from $R$ into $M$.

We will often write copure Baer injective and mean $\mathcal{Q}$-copure Baer injective for some given class $\mathcal{Q}$, just like when we say module and homomorphism (or map) and mean $R$-module and $R$-homomrphism (or $R$-map) for some given ring $R$.

Examples. 1) Injective modules are $\mathcal{Q}$-copure Baer injective for any class $\mathcal{Q}$.

2) All pure Baer injective (and therefore all pure injective) modules are $\mathcal{P} \mathcal{I}$-copure Baer injective.

3) Putting the class $\mathcal{Q}=\{\mathbb{Z}\}$, we see that none of the proper ideals of $\mathbb{Z}$ is $\{\mathbb{Z}\}$-copure. Hence all $\mathbb{Z}$-modules are $\{\mathbb{Z}\}$-copure Baer injective but of course not all of them are injective.

4) We know that all $\mathbb{Z}$-modules are pure Baer injective, however, not all of them are $\mathcal{Q}$-copure Baer injective for all classes $\mathcal{Q}$. For example, let 
$M$ be any injective $\mathbb{Z}$-module, so that all ideals of $\mathbb{Z}$ are $\{M\}$-copure in $\mathbb{Z}$. Hence the $\{M\}$-copure Baer injective modules are precisely the injective ones. Therefore $\mathbb{Z}$, for instance, is not $\{M\}$-copure Baer injective.

5 ) Let the ring $R$ be $\mathbb{Z}_{4}$ and $\mathcal{Q}=\left\{\mathbb{Z}_{4}\right\}$. Since $\mathbb{Z}_{4}$ is quasi injective, the sequence $0 \rightarrow \mathbb{Z}_{2} \rightarrow \mathbb{Z}_{4}$ is $\mathcal{Q}$-copure exact. It is in fact the only nontrivial one! So, both of $\mathbb{Z}_{4}$ and $\mathbb{Z}_{3}$ are $\mathcal{Q}$-copure Baer injective, while $\mathbb{Z}_{2}$ is not. To see this consider the following diagram:

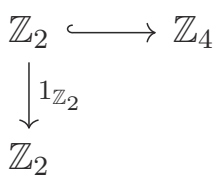

which cannot be completed because $\mathbb{Z}_{2}$ is not a direct summand of $\mathbb{Z}_{4}$.

6) If $\mathcal{Q}$ is the class of simple modules then the class of copure Baer injective modules equals the class $\mathcal{M}$ of modules injective with respect to all inclusions $I \rightarrow R$ with $I$ an $s$-pure ideal of a commutative ring $R$, see [1].

7) Any module $Q$ is, of course, $\{Q\}$-copure Baer injective but may not, in general, be pure Baer injective.

The following proposition is easy to verify.

Proposition 1. 1) The direct product (resp., direct sum) of a (finite) family of modules is copure Baer injective if and only if each factor is copure Baer injective.

2) An R-module $M$ is copure Baer injective if and only if $\operatorname{Ext}(R / I, M)=0$ for every copure left ideal $I$ of $R$.

Proposition 2. The class of copure Baer injective modules is closed under extensions.

Proof. Let $0 \rightarrow A \rightarrow B \rightarrow C \rightarrow 0$ be an exact sequence with $A$ and $C$ copure Baer injective. Exactness of the sequence $0 \rightarrow \operatorname{Ext}(R / I, A) \rightarrow$ $\operatorname{Ext}(R / I, B) \rightarrow \operatorname{Ext}(R / I, C) \rightarrow 0$ gives, by Proposition 1, that $\operatorname{Ext}(R / I$, $B)=0$ for any copure left ideal $I$ of $R$.

Thani [3] introduced left pure hereditary rings as those rings whose every pure left ideal is projective. Here, we define left copure hereditary rings.

Definition 2. The ring $R$ is called left $\mathcal{Q}$-copure hereditary if every copure left ideal of $R$ is projective. 
Of course, left pure hereditary rings are $\mathcal{P} \mathcal{I}$-copure hereditary. We will just say 'left copure hereditary' when the class $\mathcal{Q}$ is known.

Theorem 1. The following statements are equivalent:

1) The ring $R$ is left copure hereditary.

2) The homomorphic image of any copure Baer injective R-module is copure Baer injective.

3) The homomorphic image of any injective $R$-module is copure Baer injective.

4) Any finite sum of injective $R$-modules is copure Baer injective.

Proof. (1) $\Rightarrow$ (2) Consider the diagram

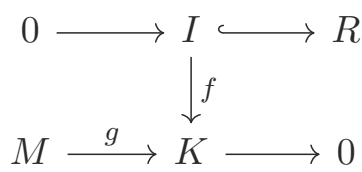

of $R$-modules, where $I$ is a copure left ideal in $R$ and $M$ is a copure Baer injective module. Projectivity of $I$ gives the existence of a $\phi: I \rightarrow M$ such that $g \phi=f$. Copure Baer injectivity of $M$ gives a map $\phi^{\prime}: R \rightarrow M$ extending $\phi$, hence $g \phi^{\prime}$ extends $f$ and $K$ is copure Baer injective. $(2) \Rightarrow(3)$ is trivial. $(3) \Rightarrow(1)$ Let $I$ be a copure left ideal of $R$ and consider the following diagram for a given $R$-module $M$ :

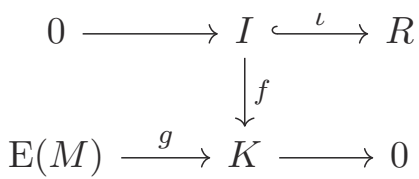

where $\mathrm{E}(M)$ denotes the injective envelope of $M$. Since $K$ is copure Baer injective, there is a map $h: R \rightarrow K$ such that $\left.h\right|_{I}=f$. Projectivity of $R$ gives a $\sigma: R \rightarrow E(M)$ such that $g \sigma=h$, i.e. $g \sigma \iota=h \iota=f$. This means $I$ is $\mathrm{E}(M)$-projective, i.e. $I$ is projective. $(3) \Rightarrow(4)$ is clear. (4) $\Rightarrow(3)$ Similar to the proof of $(4) \Rightarrow(3)$ in $[3$, Theorem 2.2].

\section{Embedding in copure baer injective modules}

The main result of this section is the following:

Theorem 2. Let $\mathcal{Q}$ be a non-empty class of $R$-modules. Every module can be embedded as a $\mathcal{Q}$-copure submodule in some $\mathcal{Q}$-copure Baer injective module. 
We break the proof into the following three lemmas.

Lemma 2. Every module can be embedded in a copure Baer injective module.

Proof. Given a module $A$, we want to show the existence of a copure Baer injective module that contains $A$ as a submodule. Consider the copure left ideals $I$ of $R$ and the set $\mathscr{F}$ of all maps $f: I \rightarrow A$. Thus, for any $f \in \mathscr{F}$ there is a pushout $B$ and a map $g: R \rightarrow B$ with $\left.g\right|_{I}=f$. The module $B$ may not be copure Baer injective, so put $A_{0}=A, A_{1}=B$ and repeat the above process with $A$ replaced by $A_{1}$ to give $A_{2}$ and $A_{0} \subseteq A_{1} \subseteq A_{2}$. Continuing in this manner, we get a sequence $A_{0} \subseteq \cdots \subseteq A_{n} \subseteq A_{n+1} \subseteq \cdots$, for all $n \in \mathbb{N}$. Put $A_{\omega}=\bigcup A_{n}$. Now, for each nonlimit ordinal repeat the above process. If we get to a limit ordinal, say $l$, define $A_{l}=\bigcup\left\{A_{s}, s<l\right\}$. Let $t$ be the smallest ordinal with cardinality bigger than that of the ring $R$, i.e. $|t|=|R|^{+}$(the successor cardinal of $|R|$ ). For each $s<t$, we have $|s|<|t|, t$ is an initial ordinal and $A_{t}=\bigcup\left\{A_{s}, s<t\right\}$. Now, $A_{t}$ is our copure Baer injective module. To see this, let $I$ be a copure left ideal of $R$ and $f: I \rightarrow A_{t}$ any map. For each $r \in I$, let $s(r)$ be the smallest ordinal such that $f(r) \in A_{s(r)}$. Then $s(r)<t$ and $|s(r)|<|t|=|R|^{+}$. Hence $|s(r)| \leqslant|R|$. Put $p=\sup \{s(r), r \in R\}$. As each $|s(r)| \leqslant|R|$, we must have $|p| \leqslant|R|<|t|$. Hence, $p<t$. Since $t$ is a limit ordinal, we have $p+1<t$. Therefore, for each $r \in R, r \in A_{s(r)} \subseteq A_{p} \subseteq A_{p+1} \subseteq A_{t}$. So, $f(I) \subseteq A_{p}$. Moreover, the map $f: I \rightarrow A_{p}$ can be extended to a map $g: R \rightarrow A_{p+1}$ with $\left.g\right|_{I}=f$. View $g$ now as a map $R \rightarrow A_{t}$. (The proof is adapted from [2, p. 295].)

Of course, we know that every module can be embedded in an injective (hence copure Baer injective) module. But this, unlike the next lemmas, does not guarantee that the embedding is copure.

Lemma 3. Suppose that $A_{0} \subseteq A_{1} \subseteq \cdots$ is an ascending chain of modules such that $A_{i}$ is a copure submodule of $A_{i+1}$ for all $i$. Then, $A_{0}$ is copure in $\bigcup A_{i}$.

Proof. Let $M$ be a member of the class $\mathcal{Q}$ and $f_{0}: A_{0} \rightarrow M$ a map which extends, by assumption, to a map $f_{1}: A_{1} \rightarrow M$, which in turn extends to $f_{2}: A_{2} \rightarrow M$, and so on. View the maps $f_{i}$ as sets of ordered pairs $\left(a_{i}, f\left(a_{i}\right)\right)$ with $a_{i} \in A_{i}$ for all $i$. Hence, it is clear that $f_{i} \subseteq f_{i+1}$ for all $i$ and if $\left(x, y_{1}\right),\left(x, y_{2}\right) \in f_{i}$ for some $i$ then $y_{1}=y_{2}$. Now, claim that $f=\cup f_{i}$ is a (well-defined) homomorphism. To see this, let $x \in \cup A_{i}$, i.e. $x \in A_{i}$ for some $i$ and $\left(x, f_{i}(x)\right) \in f_{i} \subseteq f$. If $\left(x, y_{1}\right),\left(x, y_{2}\right) \in f$, then 
$\left(x, y_{1}\right) \in f_{i}$ and $\left(x, y_{2}\right) \in f_{j}$ for some $i$ and $j$. Without loss of generality, assume $i \leqslant j$, so that $\left(x, y_{1}\right)$ and $\left(x, y_{2}\right)$ are both in $f_{j}$ and therefore $\left(x, y_{1}\right)=\left(x, y_{2}\right)$ and $f$ is well-defined. To finish the proof, let $x, y \in \cup A_{i}$ so that $x \in A_{i}$ and $y \in \cup A_{j}$ for some $i$ and $j$. Again assume $i \leqslant j$, so $f_{j}(x)=f_{i}(x)$. Now, for any $r, s \in R, f_{j}(r x+s y)=r f_{j}(x)+s f_{j}(y)$. So $f(r x+s y)=r f(x)+s f(y)$.

Lemma 4. The embedding in Lemma 2 is copure.

Proof. The construction of $A_{i}$ in Lemma 2 shows, by (1) of Lemma 1, that $A_{i}$ is copure in $A_{i+1}$ for all $i$, and by Lemma $3, A$ is copure in $\bigcup A_{n}=A_{\omega}$. Again by Lemma $3, A_{\omega}$ is copure in $A_{\omega+1}$ and $A_{\omega+1}$ is copure in $A_{\omega+2}$ and so on. In other words, for every ordinal $s<|R|^{+}$, we have either $A$ is copure in $A_{s}$ if $s$ is not a limit ordinal, or $A_{s}=\bigcup_{u<s} A_{u}$ if $s$ is a limit ordinal. In either case, $A$ is copure in $A_{t}$, as desired.

The embedding Theorem can be used in characterizing some copure exact sequences.

Theorem 3. The sequence $0 \rightarrow I \stackrel{\iota}{\hookrightarrow} R \rightarrow R / I \rightarrow 0$ is copure exact if and only if every copure Baer injective $R$-module is injective with respect to it.

Proof. Necessity is clear. To prove sufficiency, let $j: I \rightarrow C$ be a copure embedding in a copure Baer injective module $C$ (Theorem 2). Therefore, there exists a map $f: R \rightarrow C$ such that $f \iota=j$. But $j$ is a copure monomorphism, so by (2) of Lemma $1 \iota$ is a copure monomorphism.

\section{Characterization of rings using copure Baer injectivity}

Thani [3] proved that for a left self injective ring $R$, the condition that $R / I$ is pure Baer injective for every essential left ideal $I$ of $R$ is enough to make $R / I$ pure Baer injective for all left ideals $I$ of $R$. Using the same line of argument, we generalize this to copure Baer injectivity.

Proposition 3. Let $R$ be a left self injective ring. If $R / J$ is copure Baer injective for any essential left ideal $J$ of $R$, then $R / I$ is copure Baer injective for any left ideal $I$ of $R$.

Proof. Since $R$ is injective, the injective envelope $\mathrm{E}(I)$ of $I$ must be a direct summand of $R$, for any left ideal $I$ of $R$. Therefore, $\mathrm{E}(I)=R e$ for some idempotent $e \in \mathrm{E}(I)$. Now for the map $f: R \rightarrow R e$, defined by 
$1 \mapsto e$, since $I$ is essential in $R e, f^{-1}(I)$ must as well be essential in $R$ and, therefore by assumption, $R / f^{-1}(I)$ is copure Baer injective. Define $\bar{f}: R e / I \rightarrow R / f^{-1}(I)$ by $r e+I \mapsto r+f^{-1}(I)$ and proceed as in the proof of [3, Proposition 2.3].

By a left $\mathcal{Q}$-copure split ring we mean a ring every $\mathcal{Q}$-copure left ideal of which is a direct summand (hence a principal ideal). Clearly, every left pure split ring is left $\mathcal{P} \mathcal{I}$-copure split and if a ring $R$ is left copure-split then it is left copure hereditary. The $\mathcal{Q}$-copure split rings are characterized in the following Theorem.

Theorem 4. The following statements are equivalent:

1) The ring $R$ is left copure split.

2) Every $R$-module is copure Baer injective.

3) Any copure left ideal of $R$ is copure Baer injective.

4) (a) $R$ is left copure hereditary, and

(b) Every free left $R$-module is copure Baer injective.

Proof. (1) $\Rightarrow(2)$ Let $M$ be an $R$-module. Since every left ideal $I$ of $R$ is a direct summand, every map $I \rightarrow M$ into any $R$-module can easily be extended to a map $R \rightarrow M$. (2) $\Rightarrow(3)$ is obvious. (3) $\Rightarrow(1)$ Let $I$ be a copure left ideal of $R$. Copure Baer injectivity of $I$ gives a homomorphism $R \rightarrow I$ that extends the identity map of $I$, which means $I$ is a direct summand of $R$. (1) $\Rightarrow(4)(\mathrm{a})$ and $(2) \Rightarrow(4)(\mathrm{b})$ are immediate. (4) $\Rightarrow(3)$ Let $I$ be a copure left ideal of $R$, hence projective by (a) and therefore a direct summand of some free $R$-module $F$. From (b) it follows that $F$ is copure Baer injective and by Proposition 1, so is $I$.

Recall that a ring $R$ is called left coregular if every left ideal of $R$ is copure in $R$ [4, p.324].

Theorem 5. For a ring $R$ the following statements are equivalent:

1) The ring $R$ is left coregular.

2) Every copure Baer injective R-module is injective.

3) Every copure Baer injective R-module is quasi injective.

Proof. (1) $\Rightarrow(2) \Rightarrow(3)$ are obvious. (2) $\Rightarrow$ (1) By assumption, every copure Baer injective $R$-module is injective with respect to any sequence $0 \rightarrow I \rightarrow R \rightarrow R / I \rightarrow 0$, which must, therefore, be copure exact by Theorem 3. (3) $\Rightarrow(2)$ Let $M$ be a copure Baer injective $R$-module. Hence, by Proposition 1, so is $M \oplus \mathrm{E}(R)$ which must be quasi injective by assumption. Therefore, $M$ is injective with respect to $\mathrm{E}(R)$. In particular, $M$ is $R$-injective or injective by Baer condition. 


\section{References}

[1] Iuliu Crivei, s-Pure submodules, Int. J. Math. Math. Sci. 2005, 2005, pp.491-497.

[2] John Dauns, Modules and Rings, Cambridge University Press, 1994.

[3] Nada M. Al Thani, Pure Baer injective modules, Int. J. Math. Math. Sci. 20, 1997, pp.529-538.

[4] Robert Wisbauer, Foundations of Module and Ring Theory. Gordon and Breach, 1991.

\section{CONTACT INFORMATION}

Mohanad Farhan Department of Production Engineering and Hamid Metallurgy, University of Technology-Iraq, Baghdad, Iraq

$$
\begin{aligned}
E-M a i l(s): & 70261 @ u o t e c h n o l o g y . e d u . i q, \\
& \text { mohanadfhamid@yahoo.com }
\end{aligned}
$$

Received by the editors: 30.06 .2018 . 